\title{
BIOLOGIE DE TACHINA LARVARUM MEIGEN
}

\author{
Par Robert MATTHEY,
}

Docteur ès sciences

En juillet-août, je trouve assez fréquemment, sur les épilobes (Epilobium parviflorium).bordant les fossés, les chenilles de deux lépidoptères sphingidés : Chærocampa elpenor et Pterogon proserpina. Ces deux grandes chenilles, l'une brune et l'autire indifféremment verte ou brune, sont souvent parasitées par un diptère, la Tachina larvarum, mouche à peine plus grosse que Musca domestica et facilement reconnaissable à ses gros yeux rouges et à son abdomen gris cendré et noir.

Dans ma région, le nombre d'espèces parasitées par ce diptère paraît se borner aux deux Sphingidæ cités plus haut. Des lépidoptères très voisins ne me l'ont jamais fourni : c'est ainsi ıqu'en 1923 j'ai disséqué 7 chenilles de Deilephila euphorbiæ qui toutes étaient indemnes, alors qu'en 1920, j'ai observé trois cas d'infection sur 5 Chærocampa elpenor, en 1922, 6 cas sur 15 exemplaires de Pterogon proserpina, et, en 1923,8 cas sur 17 individus de la même espèce.

D'autres papillons crépusculaires (Dilina tiliæ, Sphinx ligustri, Hyloicus pinastri), examinés au hasard de mes trouvailles, ne m'ont jamais procuré le diptère parasite.

En disséquant une chenille adulte et infectée on constate tout d'abord la disparition presque complète du corps adipeux (fig. 1). Une observation plus attentive montre les larves de Tachina, implantées chacune dans une sorte de " cornet " chitineux dont la partie évasée, presque transparente, reçoit leur extrémité postérieure et dont la partie rétrécie, fortement chargée de chitine s'ouvre à la face interne des stigmates de la chenille. Le résultat de ce dispositif est évident : la respiration des parasites est assurée puisque leurs orifices respiratoires, situés postérieurement, reçoivent d'une façon immédiate l'air qui pénètre par les stigmates de leur hôte.

Un autre résultat de cet ingénieux mécanisme respiratoire est celui-ci : les larves de Tachina ne peuvent pas se déplacer à l'intérieur de la chenille et ne peuvent se nourrir que de la partie du tissu adipeux se trouvant dans leur voisinage immédiat et c'est bien ce que l'on constate en disséquant une chenille peu infectée (3-5 larves) ; la nappe adipeuse est intacte dans tous le corps de l'hôte,

Annales de Parasitologie, $\mathrm{T}$. II, $\mathrm{N}^{\circ} 3$. - Juillet 1924, p. 202. 
excepté au niveau des stigmates occupés. Au contraire, lorsque l'infection est forte, les orifices respiratoires sont presque tous pourvus d'une larve parasite, et la nappe graisseuse disparue. Le cas le plus fréquent est la présence de 8 à 12 larves implantées symétriquement dans les stigmates moyens, du $5^{\circ}$ au $12^{\circ}$ anneau.

Je reviens à ce que j'ai appelé " cornet d'implantation ". Il n'est pas possible à l'œil nu de préciser s'il s'agit d'une production de la larve parasite ou de la chenille parasitée.

Ma première idée avait été qu'il y avait là les mues successivement emboîtées de la larve du diptère. J'ai reconnu ensuite que cette interprétation était erronée, d'abord parce que les larves de muscidés ne muent que rarement (1), et ensuite parce qu'elle n'expliquai: pas l'implantation si solide du " cornet ".

Il n'est d'ailleurs pas possible de reconnaître la nature de ce corliet par l'examen d'une chenille adulte. Cet examen nous permettra seulement une dernière observation, sur- tout si nous comparons l'anatomie de notre sujet parasité avec celle d'une autre chenille de même taille, mais indemne; chez la chenille saine, les orifices stigmatiques sont reliés entre eux par des trones trachéens parallèles au tube digestif et qui les mettent en communication les uns aves les autres;

(1) Henneguy (F.). Les Insectes. Paris, 1904.

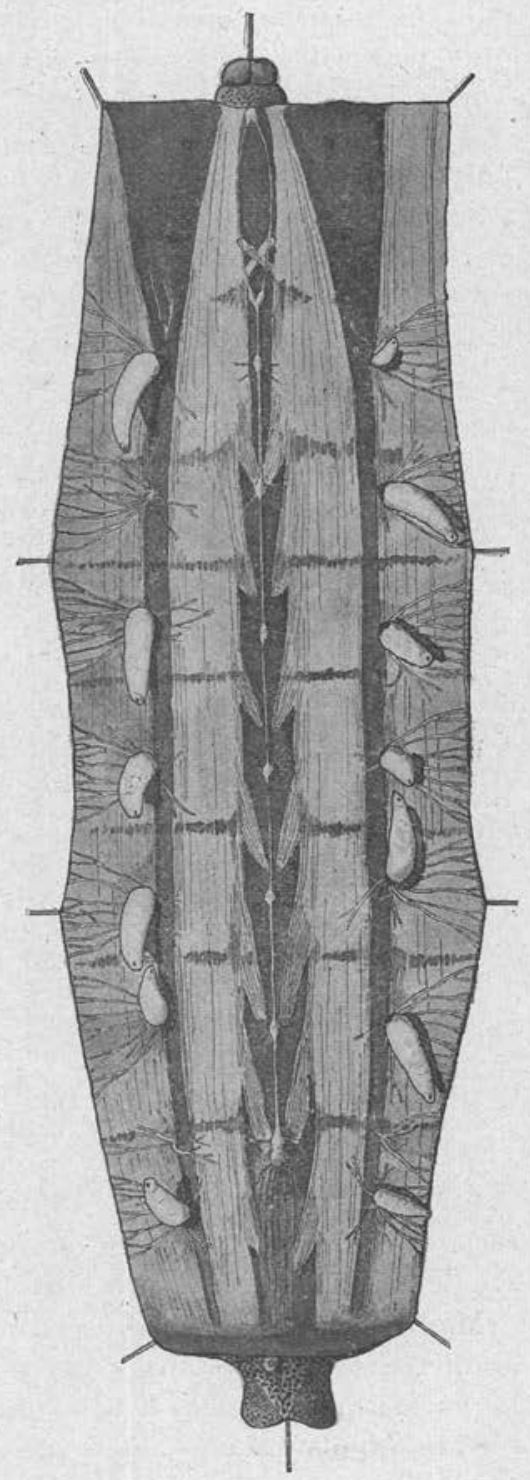

Fig. 1. - Plerogon proserpina. Chenille adulte ouverte dorsalement : le tube digestif est enlevé. 13 larves de Tachina implantées dans les stigmates sont bien visibles, ainsi que les trachées, la musculature ventrale et la chaîne ganglionnaire de la chenille, $\times 4$. 
chez la chenille parasitée, ces connectifs longitudinaux n'existent plus entre deux stigmates consécutifs occupés par des larves de Tachina.

Trois années, je ne suis pas parvenu à m'expliquer cette double énigme : formation des " cornets " et disparition des connectifs trachéens. Cette année (1923), enfin, j'ai trouvé la solution cherchée en disséquant une jeune chenille de Pterogon proserpina (elle mesurait $45 \mathrm{~mm}$., alors que prête à se transformer elle en atteint 70 ). Cette chenille ne possédait ni " cornets ", ni larves visibles, mais

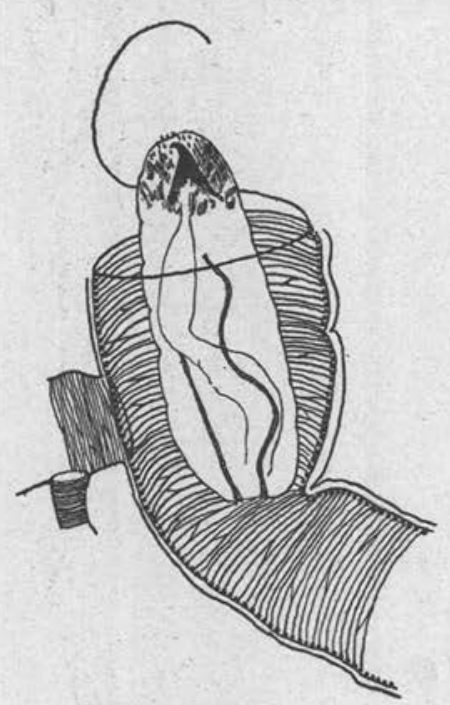

Fig. 2.- Tachina larvarum. Jeune larve engagée dans une trachée longitudinale, $\times 82$. mon attention fut attirée par l'aspect dégénéré des troncs trachéens longitudinaux. Chez une chenille saine, les trachées bourrées d'air se détachen nettement en cordons d'un blanc argenté brillant; chez le sujet que j'examinais, au contraire, les connectifs étaient jaunâtres et ternes. Je les découpai alors et les examinai sous le microscope, dans une goutte de glycérine, entre lame et lamelle. Dans un des fragments je constatais la présence d'une jeune larve de Tachina engagée à l'intérieur de la trachée (fig. 2) ; cette dernière est considérablement altérée ; la spirale de chitine, ordinairement régulière, présente de nombreuses solutions de continuité ; l'intima paraît avoir disparu. La larve elle-même diffère passablement de ce qu'elle sera comme adulte. L'armature buccale est relativement très forte et toute l'extrémité antérieure, rembrunie de chitine, est hérissée de petits tubercules saillants.

Maintenant, il est facile de concevoir son évolution ultérieure ; le diamètre de la larve augmente jusqu'à atteindre celui de la trachée. Le parasite perce alors le tube qui le renferme et commence de dévorer la nappe adipeuse ; mais il a soin de garder son extrémité postérieure, porteuse de la plaque stigmatique (fig. 3) engagée dans le connectif rompu. Celui-ci subit alors des modifications remarquables de structure et de volume. La couche chitinogène ne sécréte plus la spirale, principal caractère histologique de la trachée, spirale dont on ne retrouve plus que quelques rares vestiges (fig. 4).

Par contre, la chitine se condense en un anneau chitineux très 
robuste à l'extrémité stigmatique de l'ancien connectif en voie de devenir " cornet ". Cette métamorphose se complète par la dilatation progressive que fait subir la larve parasite au tronçon trachéen dans lequel est engagée son extrémité postérieurè. Cette dilatation a pour effet final de transformer en un cône ce qui était un cylindre ; le mécanisme histologique de ce processus m'est inconnu : je crois cependant pouvoir l'attribuer à une prolifération des cellules hypodermiques représentant la couche génératrice de la cuticule externe. J'insiste encore quelque peu sur cette adaptation qui me paraît absolument remarquable puisque, sous l'influence de la larve parasite, l'organisme réagit en fournissant à son ennemi les moyens de respirer. Cela constitue une " réaction de défense " pour le moins imprévue.

Je reviens à la larve de Tachina: bientột le tissu adipeux est consommé, et l'épuisement des vivres coïncide avec le moment où

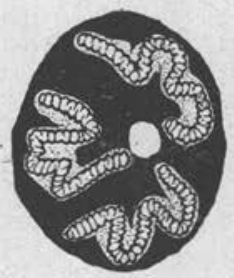

Fig. 3. - Tachina larvarum.

Plaque stigmatique de la larve, $\times 82$.

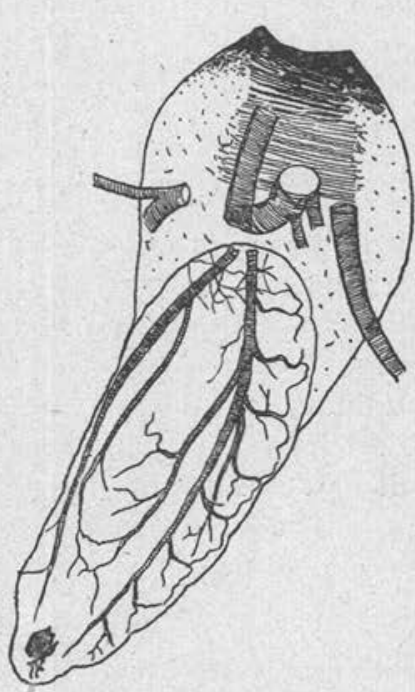

Fig. 4. - Tachina larvarum. Larve adulte daus son cornet d'implantation, $\times{ }^{*} 32$.

la chenille, inquiète, commence d'arpenter sa prison, cherchant un lieu propice à la nymphose. Celle-ci ne se produira jamais. 2 ou 3 jours la chenille se flétrit, dépérit, puis c'est la mort. Quelques heures après, les larves de Tachina perforent la peau déjà putréfiée de leur hôte et s'enfoncent en terre pour s'y transformer en une pupe semblable à celle de tous les muscidés : barillet ovoïde, couleur de terre. Cette métamorphose a lieu vers la fin de juillet et le début d'août. Le diptère adulte apparaîtra en juin-juillet l'année suivante.

Et maintenant, revenons encore une fois à la jeune larve que nous avons trouvé engagée dans un des trones trachéens longitudinaux. Comment y est-elle parvenue ? Sur une quarantaine de chenilles de Pterogon proserpina et de Chærocampa elpenor que j'ai 
examinées, je n'ai jamais trouvé les œufs de la Tachina, probablement parce que ces œufs éclosent très rapidement (1). Une autre espèce me permet de combler cette lacune : En automne qui n'a rencontré une chenille velue et brune, se roulant en anneau au moindre contact? Elle appartient à un bombyx (Hacrothylacia rubi) commun en juin-juillet. Or cette chenille porte fréquemment des œufs de diptères (mes élevages ne m'ont pas encore procuré l'espèce, sans doute une autre Tachina, ou une Echinomyia) fixés régulièrement sur les côtés de l'animal, dans la région péristigmatique. Une seule fois, j'ai trouvé un œuf placé ventralement ; jamais je n'en ai vu sur le dos.

Fabre (2) nous a montré les hyménoptères prédateurs paralyser leurs victimes au moyen de l'instillation d'une goutte de venin dans les centres nerveux de celles-ci. Sans atteindre cette exquise précision, l'ouvrage de la Tachina n'en est pas moins remarquable puisqu'elle place ses œufs dans le voisinage immédiat des stigmates.

Je n'ai pas encore vu l'éclosion, mais la logique nous montre ce que l'observation nous a refusé : La larve éclôt, trouve l'orifice stigmatique béant, y pénètre et s'engage dans les troncs trachéens longitudinaux où nous l'avons précédemment trouvée.

Les choses sont rarement aussi simples que nous le pensons : la biologie des Tachina, par exemple, ressemble d'assez loỉn à la conception grossière du parasite " perȩant la peau pour se nourrir de la nappe adipeuse de son hôte " (3).

(1) Plusieurs Entomobies sont vivipares: j'ignore s'il en est ainsi chez l'espèce qui nous occupe.

(2) Faвne (J.-H.). Souvenirs entomologiqnes, séries 1, 2, 3, 4.

(3) Lampent (K.). Die Grosschmeterlinge Mitleleuropas. 